\title{
FRET Response of a Modified Ribose Receptor Expressed in the Diatom Thalassiosira pseudonana
}

HR Miller

August 2011

Pacific Northwest

NATIONAL LABORATORY

Proudly Operated by Battelle Since 1965 


\title{
DISCLAIMER
}

This report was prepared as an account of work sponsored by an agency of the United States Government. Neither the United States Government nor any agency thereof, nor Battelle Memorial Institute, nor any of their employees, makes any warranty, express or implied, or assumes any legal liability or responsibility for the accuracy, completeness, or usefulness of any information, apparatus, product, or process disclosed, or represents that its use would not infringe privately owned rights. Reference herein to any specific commercial product, process, or service by trade name, trademark, manufacturer, or otherwise does not necessarily constitute or imply its endorsement, recommendation, or favoring by the United States Government or any agency thereof, or Battelle Memorial Institute. The views and opinions of authors expressed herein do not necessarily state or reflect those of the United States Government or any agency thereof.

\author{
PACIFIC NORTHWEST NATIONAL LABORATORY \\ operated by \\ BATTELLE \\ for the \\ UNITED STATES DEPARTMENT OF ENERGY \\ under Contract DE-AC05-76RL01830
}

Printed in the United States of America
Available to DOE and DOE contractors from the Office of Scientific and Technical Information,
P.O. Box 62, Oak Ridge, TN 37831-0062;
ph: (865) 576-8401
fax: $(865) 576-5728$
email: reports@adonis.osti.gov

\footnotetext{
Available to the public from the National Technical Information Service, U.S. Department of Commerce, 5285 Port Royal Rd., Springfield, VA 22161 ph: (800) 553-6847 fax: $(703) 605-6900$ email: orders@ntis.fedworld.gov online ordering: http://www.ntis.gov/ordering.htm
}

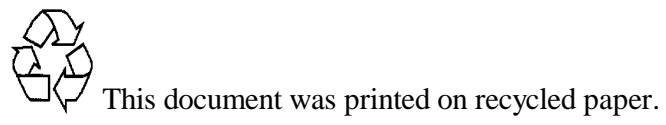




\section{FRET Response of a Modified Ribose Receptor Expressed in the Diatom Thalassiosira pseudonana}

HR Miller

August 2011

Prepared for the U.S. Department of Energy under Contract DE-AC05-76RL01830

Pacific Northwest National Laboratory

Richland, Washington 99352 




\title{
FRET Response of a Modified Ribose Receptor Expressed in the Diatom Thalassiosira pseudonana
}

\author{
Hanna R. Miller \\ Office of Science, Science Undergraduate Laboratory Internship Program \\ Michigan State University, East Lansing, Michigan \\ Pacific Northwest National Laboratory-Marine Sciences Laboratory \\ Sequim, WA
}

August 19, 2011

Prepared in partial fulfillment of the requirement of the Office of Science, Department of Energy's Science Undergraduate Laboratory Internship under the direction of G. Roesijadi and Kathryn E. Marshall, Marine Biotechnology, Environment and Energy Directorate, Pacific

Northwest National Laboratory 


\begin{abstract}
The ability to insert complex proteins into silica has many applications including biosensing. Previous research has demonstrated how to direct proteins to the biosilica of diatoms [1]. Here, we show that a complex fusion protein that includes an enzyme, a bacterial ribose periplasmic binding protein, flanked by fluorescent proteins constituting a FRET pair can remain functional in the frustules of living diatoms. A Sil3 tag is attached to the Nterminal end to localize the fusion protein to frustules of the diatom Thalassiosira pseudonana. When ribose was applied, a larger decrease in FRET response was seen in transformed cells than in untransformed cells. Multiple forms of the expression vector were tested to find the optimal system; specifically, a one-vector system was compared to a twovector system and the gDNA version of the Sil3 localization tag was compared to the cDNA version. The optimal system was found to be a one-vector system with the genomic version of the Sil3 tag to direct the protein to the frustules. Localization of the enzyme to the frustules was further confirmed through cell fluorescence imaging.
\end{abstract}

\title{
INTRODUCTION
}

Silica is emerging as a platform on which to better conduct sensing, organic synthesis, remediation, and drug delivery [1]. Although the final goal of these emerging technologies is different, they all rely on the immobilization of proteins in the silica matrix. Incorporation of complex proteins into silica presents potential problems as the harsh procedures required for physical insertion often denature the proteins of interest. A recently explored solution to overcome this utilizes organisms capable of producing highly structured biosilica matrices, forming platforms of higher quality than capable through abiotic procedures [2]. Diatoms, a unicellular eukaryote found in many freshwater and marine environments, are one of these organisms as they form intricate biosilica cell wall called frustules.

Recently, a method for transforming the diatom Thalassiosira pseudonana with recombinant proteins has been developed using microparticle bombardment [3]. By modifying the expression cassette with a biosilica-targeting tag (primarily using the silaffin Sil3 genomic 
sequence), transformed diatoms direct the protein of interest to the frustules [1]. This presents many opportunities to develop biosensors by immobilizing enzymes in the diatom biosilica.

To test this concept, we used the bacterial periplasmic ribose binding protein (RBP) flanked with the Förster Energy Resonance Transfer (FRET) fluorescent protein pair, CyPet (cyan) and YPet (yellow) [4]. Changes in FRET serve as the readout to determine whether or not ribose is bound to the receptor. When ribose is not bound, the two fluorescing proteins are spatially close, allowing for the energy transfer to occur. The binding of ribose changes the receptor conformation, moving the fluorescing proteins farther apart, thereby reducing the energy transfer. The ribose sensor is being used as proof-of-principle for transforming diatom biosilica into biosensors and could be replaced with any protein-based sensor in the diatom system.

Vectors containing the expression cassette CyPet-RBP-YPet (CRY) tagged N-terminally with the Sil3 tag were available from prior work at the Pacific Northwest National Laboratory, Sequim, WA (Marshall et al., unpublished work). Two versions of the Sil3 tag had been developed and are currently being used: the genomic version (gDNA) and complementary gene version (cDNA). Additionally, two different vector systems can be used to transform the diatoms: a two-vector system that contains the Sil3-CRY expression system and the antibiotic resistance on separate vectors and the one-vector systems that contains both expression cassettes on a single vector. A single vector with the cDNA encoding the Sil3 tag and the antibiotic resistance gene was not initially available. Nourseothricin resistance is used as the antibiotic resistance selection tool to isolate transformed cells. The purpose of my project was to construct the single vector system using the cDNA encoded Sil3 and test the various expression constructs 
in transformed diatoms. We compared the FRET response in cells transformed with a one-vector system to a two-vector system, as well as compared the FRET response of cells transformed with the gDNA and cDNA versions of the Sil3 tag. The optimal vector construct would then be used in future experiments.

\section{MATERIALS AND METHODS}

Construction of cDNA one-vector system. Our lab recently created a vector containing the periplasmic ribose binding protein (RBP) flanked by the FRET pair CyPet and YPet tagged Nterminally with either the genomic (gDNA) or the complimentary gene (cDNA) version of the Sil3 biosilica targeting sequence resulting in a Sil3-CyPet-RBP-YPet (Sil3-CRY) sensing cassette. The Sil3-CRY cassette was driven by the constitutively active promoter fucoxanthin chlorophyll a/c binding protein ( $f c p$ ) (Fig. 1). Originally, the antibiotic selection gene was expressed on a second vector, $p T p f c p / n a t$, encoding the gene for nourseothricin resistance. We combined the two vectors so the selection marker and the expression cassette were on one vector. The $p T p f c p /$ nat sequence was first amplified by PCR, yielding a $2.3 \mathrm{~kb}$ product. The purified pTpfcp/nat sequence was used as a primer in overlap extension PCR to insert the antibiotic resistance cassette into the cDNA version of the Sil3-CRY (Sil3c-CRY) vector to form an approximate $9 \mathrm{~kb}$ vector.

We screened E. coli colonies transformed with the resulting $p T p f c p / S i l 3 c-C R Y: f c p / n a t$ PCR product for the $p T p f c p / n a t$ incorporation by colony PCR. Colonies containing the correct band size of $2.3 \mathrm{~kb}$ were grown over night in LB broth plus ampicillin. A Qiagen mini prep kit was used according to manufacturer's instructions to extract the vector DNA. Restriction enzyme digest with Kpn1 was used to further identify the colonies containing the correctly amplified 
vector. Digested samples producing the band sizes of 3400, 2450, $3300 \mathrm{~kb}$, and $800 \mathrm{~kb}$ were sequenced for the nat gene. Sequencing results identified the correct vector to be used in future diatom transformations for a one-vector system (Fig.1).

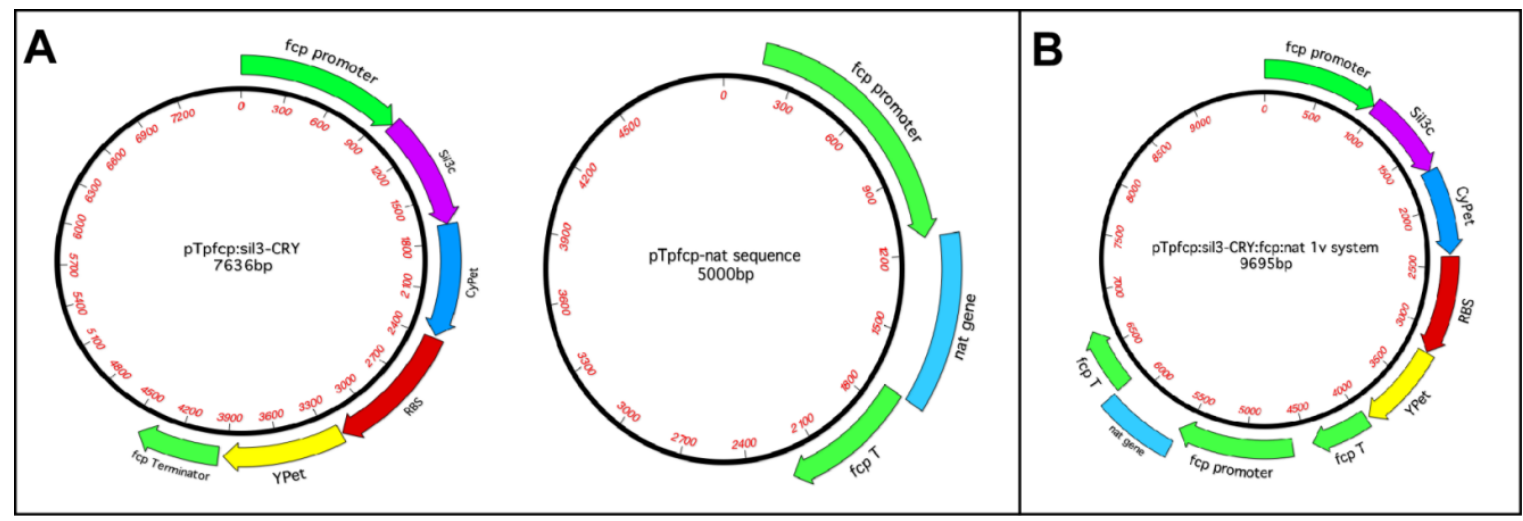

Figure 1. Vector Constructs Used in Diatom Transformations. A. Two-vector system with the Sil3-CyPet-RBP-YPet (Sil3-CRY) sensing cassette on a separate vector than the nourseothricin resistance. B. Single vector system with both expression cassettes on the same vector ( $p T p f c p / S i l 3 c-C R Y: f c p / n a t)$.

Diatom Transformation. Transformation of diatoms with the appropriate vector was accomplished using microparticle bombardment. The same procedure was used for diatom transformation of the one- and two-vector systems. DNA was prepared and transformed into $T$. pseudonana following the protocol outlined in Poulsen et al., 2006 [3]. Transformed cells were identified by their resistance to nourseothricin and biosilica-localized cyan and yellow fluorescence expression.

FRET Analysis. The change in FRET was determined after the addition of ribose to both transformed (TR) and untransformed (WT) cells. One hundred microliters of test culture was added to the well in a collagen-coated glass bottom culture dish (MatTek Corp.) and left undisturbed for at least $30 \mathrm{~min}$ to adhere the cells to the collagen. Unbound cells were washed 
away and $100 \mathrm{ul}$ of deionized water was added to the well. Cell images were captured with a Cool SNAP EZ camera containing a duel image splitter with cyan and yellow filters. Cells were photobleached for five minutes to reduce non-ribose dependent variability in FRET during the first few minutes of testing. Simultaneous cyan and yellow fluorescence images were captured every minute over the course of the experiment to determine the FRET response. After 5 min of imaging to establish a baseline FRET response, ribose was added to the cells and images were captured for an additional 10 min to determine the maximum FRET reduction and record any recovery of the cell.

The CyPet and Ypet relative fluorescence units (RFU) for a given cell were measured using the Metamorph Software. The FRET ratio was determined by dividing YPet RFU by CyPet RFU and removing the background fluorescence from each spectrum. The average FRET response was found before ribose addition and compared to the minimum ratio found during the first 3 min following the addition of ribose. On average, cells began to recover from ribose addition after $3 \mathrm{~min}$.

A dose response was also preformed with the genomic $p T p f c p / S i l 3-C R Y: f c p / n a t$ TR and WT cultures to determine the sensitivity of transformed diatom cells to ribose. The $\Delta \mathrm{r}_{\max }$ (difference between the average FRET response before ribose addition and the minimum FRET response after) for concentrations ranging between $0.003 \mathrm{mM}$ and $300 \mathrm{mM}$ was determined and saturation curves were plotted to determine the $K_{d}$ of the construct.

Confocal Microscope. The confocal microscope at University of Washington (Keck Microscopy Facility) was used to analyze WT, gDNA-TR, and cDNA one-vector system TR cells. Cyan, 
yellow, and red fluorescent images were captured to compare recombinant protein expression (cyan and yellow) to chloroplast auto-fluorescence.

\section{RESULTS}

$\underline{\text { FRET Analysis }}$

We analyzed of the FRET response of WT cells, cDNA one-vector system, cDNA twovector system, and gDNA one-vector TR cells to determine how the constructs functioned in living diatom cells. Individual cells from each transformation condition were analyzed over time to determine the $\%$ change in FRET response between pre-and post-ribose addition. Ribose addition was found to increase the background YPet and CyPet RFU; therefore, the background intensity was subtracted out prior to calculating the FRET ratio. Photobleaching the cells for 5 min prior to beginning the experiment ensured a consistent initial FRET response. Overall, a decrease in the FRET response was seen in all cells analyzed including WT; however, the percent decrease varied significantly. The FRET response curves in all TR cells were consistent with an initial decrease followed by a small recovery (Fig. 2). After ribose addition, the TR cells took approximately $3 \mathrm{~min}$ to fully respond to the ribose addition and yield the lowest FRET value before beginning recovery. The $\%$ difference was calculated by dividing the $\Delta \mathrm{r}_{\max }$ (the average of the FRET responses 5 min prior to adding ribose minus the lowest FRET response in the 3 min following ribose addition) by the average FRET response prior to ribose addition. 


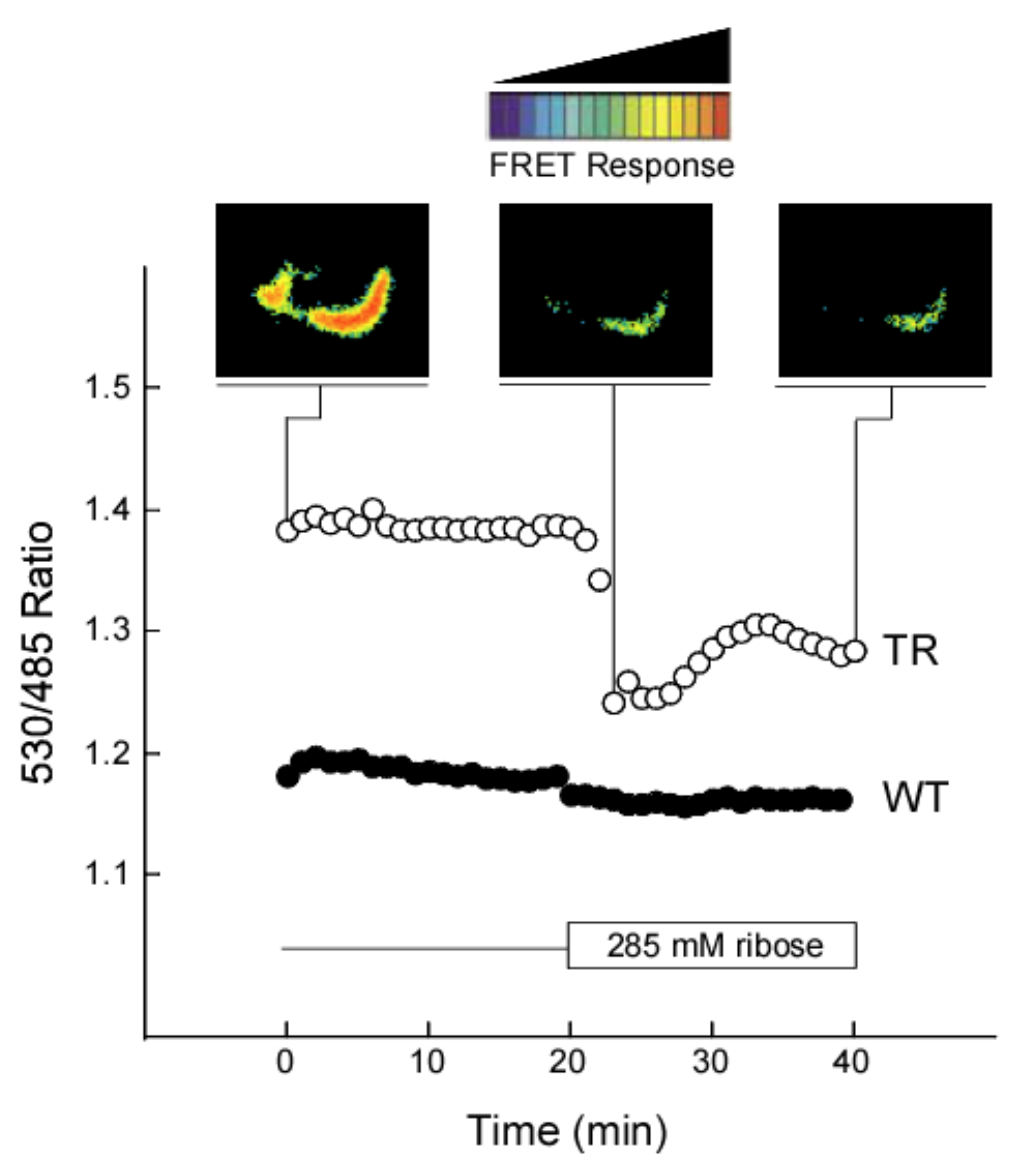

Figure 2. Average FRET Response of TR and WT Cells to Ribose. The ratio of the yellow fluorescence $(540 \mathrm{~nm})$ to cyan fluorescence $(485 \mathrm{~nm})$ was determined at one-minute intervals. Ribose addition occurred at $20 \mathrm{~min}$. Images with the magnitude of FRET response were also taken with each measurement to verify the FRET response.

FRET analysis in WT cells. We analyzed the FRET response of WT cells, using the same analysis procedure as above. WT cells had an average $6.6 \%$ decrease in a FRET-like response following ribose addition, but the response did not form the characteristic FRET response curve as seen in the TR cells. Therefore, we attributed this decrease to a background cell response.

One-Vector vs. Two-Vector System Comparisons. Past research with diatom transformations successfully used a two-vector system to introduce a gene of interest and an antibiotic resistance 
into one cell [3]. We wanted to determine whether this was an efficient transformation method to use with a FRET receptor-based system or if a one-vector system should be considered instead. In our results, the two-vector system produced a variable FRET response with an average of $6.3 \% \pm 5.2$ and a range from $3 \%$ to $17 \%$. Upon further inspection of the FRET results, the large range was due to what appeared to be the formation of two subpopulations within the data set, with some experiments creating the characteristic FRET curve and others creating curves more typical of WT cells. From the \% changes, we were able to discern two distinct groupings of cells (Fig. 3). These two groupings were found to be statistically different with one subgroup displaying a $\%$ change close to WT cells at $5.1 \% \pm 1.4$ and another subgroup of cells displaying a characteristic $\%$ change close to TR cells of $12.2 \% \pm 2.1$ ( $p$-value $<0.05)$. This difference is most likely due to the nature of the transformation. We were not able to successfully isolate single colonies on agar plates; therefore, there was large uncertainty as to whether all cells in the culture contained both vectors from the two-vector bombardment system. It is possible for some cell to possess both vectors and others to have only the antibiotic resistance

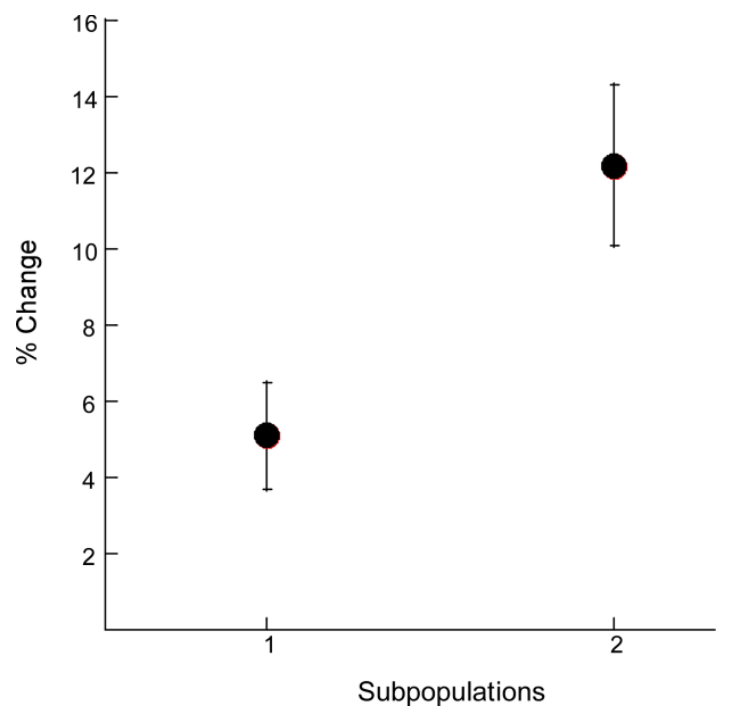


Figure 3. Subpopulations Found in the Two-Vector System. Cells transformed with the twovector system were found to either have a response typical of wild type (subpopulation 1) or transformed cells (subpopulation 2).

When examining the FRET response from a one-vector system, there was less variability in the FRET response of TR cells. TR cells displayed an average FRET decrease of $11.4 \% \pm 3.3$ with one population of FRET responses as compared to the two subpopulations that formed in the two-vector transformations. This suggests a one-vector should be used in diatom transformations so that all cells resistant to antibiotics also contain the expression cassette needing to be analyzed.

gDNA vs. cDNA Sil3 Construct Comparison. The genomic version of the Sil3 sequence was initially used to create our expression vector [4]; however, we wanted to determine if the gDNA version would produce a different FRET response compared to the cDNA version of Sil3. Diatom cells were transformed with the one-vector system of both versions and the FRET response was determined. FRET results from cells transformed with the cDNA version of the construct were found to be not statistically different from the gDNA version $(11.4 \% \pm 3.3$ vs. $11.2 \% \pm 1.0$, respectively; Fig. 4), although the cDNA version exhibited greater variability. This was expected because previous studies found the Sil3 tag does not affect the binding of ribose to the receptor. Because gDNA and cDNA FRET response was similar, we examined the fluorescence localization to determine if the Sil3-CRY tracked differently. 


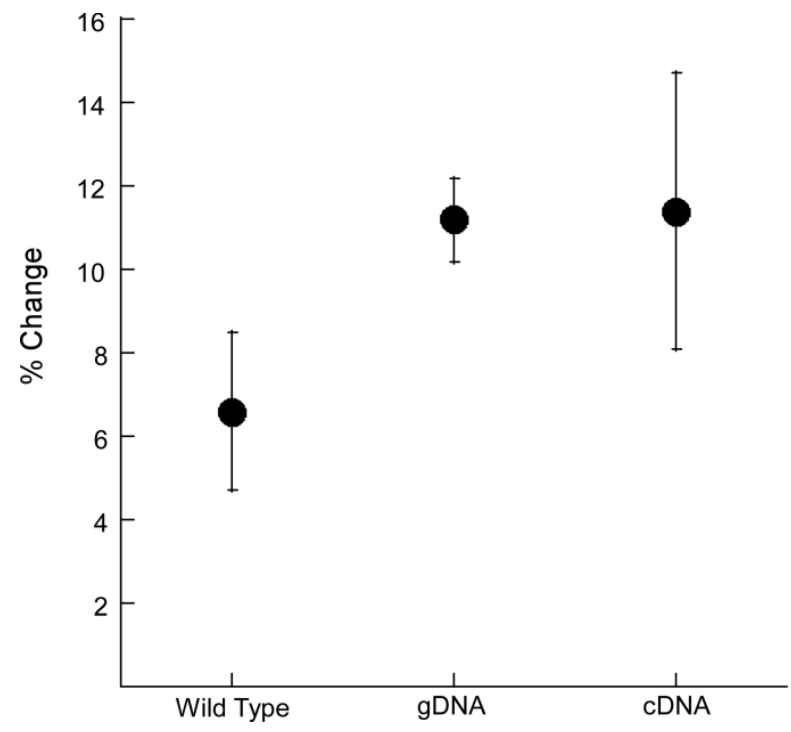

Figure 4. FRET Response Comparisons of gDNA and cDNA versions of $p$ Tpfcp/Sil3-

CRY:fcp/nat to WT Cells. The FRET response of TR cells transformed with the one-vector system containing the gDNA and cDNA version of Sil3 were compared to wild type to determine if the various Sil3 sequences affect the FRET response of the sensor.

\section{Fluorescence Localization}

Cyan and yellow fluorescence expression was analyzed in gDNA and cDNA TR cells and in WT cells by confocal microscopy in order to determine whether or not the fluorescence was localized to the frustules and how much of the YPet and CyPet fluorescence could be attributed to chloroplast bleed through. The yellow and cyan images were overlaid with images displaying the red auto-fluorescence from the chloroplasts. In the gDNA TR cells, we found large portions of the CyPet to be outlining an area on the edges of the cell suggesting the presence of the receptor in the biosilica (Fig 5). Yellow tended to co-localize with the red autofluorescence of the chloroplasts suggesting either expression of the construct is present in the chloroplasts, or the bright red auto-fluorescence in bleeding into the yellow channel. There appears to be a faint yellow outline of the cell but it is overpowered by the chloroplast-yellow 
co-localization. Both the YPet and CyPet fluoresced much brighter in the TR cells when compared to the WT in the chloroplast region; therefore, not all of the fluorescence co-localizing with the chloroplasts can be attributed to bleed-through. This fluorescence may be due to targeting of the receptor to the chloroplasts in addition to the frustules. Overall, the higher intensity of the YPet and CyPet fluorescence in TR cells signifies the presence of our sensor protein.

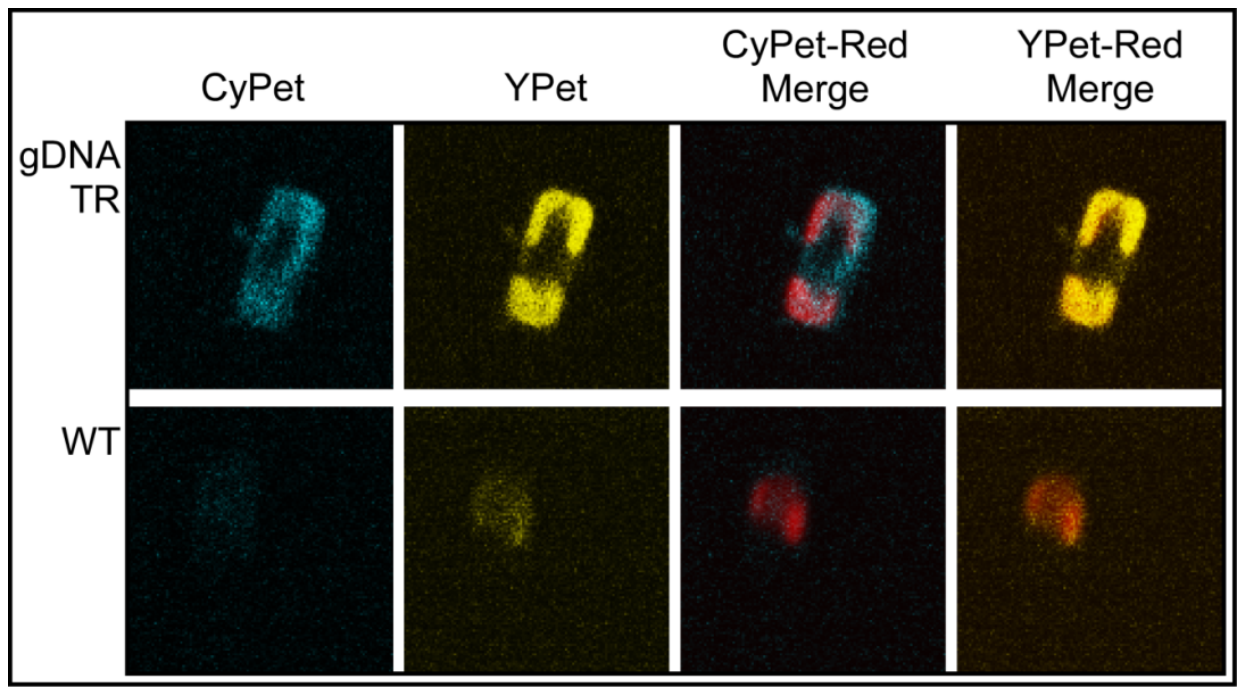

Figure 5. Cyan and Yellow Fluorescence Images of gDNA TR and WT Cells. The red autofluorescence from the chloroplasts was overlaid on the cyan and yellow fluorescence images to determine the localization of the ribose receptor. Images were captured using confocal microscopy.

When we analyzed images of the cDNA one-vector TR cells, we noticed some variations in the localization patterns. Overlaying the CyPet image with the chloroplasts showed large regions of bright fluorescence that did not line up with the chloroplasts or the frustules. This was not seen in the YPet image. The YPet fluorescence was considerably dimmer compared to what was seen in the gDNA cells and only slightly brighter than seen in the WT cells (Fig. 7). The cell 
may not be correctly processing the cDNA version, causing localization of the Sil3c-CRY protein to be targeted to a different part of the cell. These images (Fig. 6) may explain the larger variability in FRET ratio of these cells (Fig. 4).

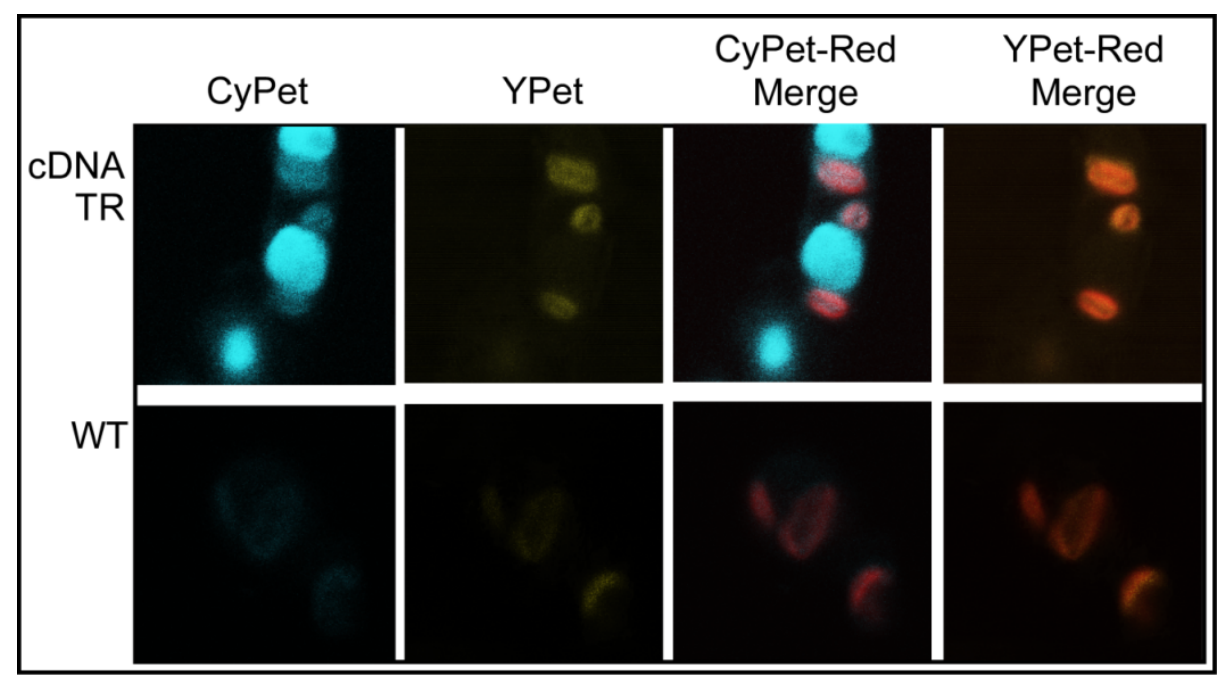

Figure 6. Cyan and Yellow Fluorescence Images of cDNA TR and WT Cells. CyPet and YPet images merged with the red fluorescence from the chloroplasts show the localization of the fluorescence. Confocal microscopy was used for cell imaging.

\section{$\underline{\text { Dose Response }}$}

Since we found the gDNA one-vector system to produce the most consistent FRET response, we used this established cell line to determine the detection limit of the sensor construct in living diatoms. We ran a 10-fold dilution series of ribose treatments in TR cells starting at $300 \mathrm{mM}$ down to $0.003 \mathrm{mM}$. Typical TR \% change of the generated FRET responses were found with the addition of $300 \mathrm{mM}$ and $30 \mathrm{mM}$, while lower concentrations resulted in a more typical WT \% change (Fig. 7). 

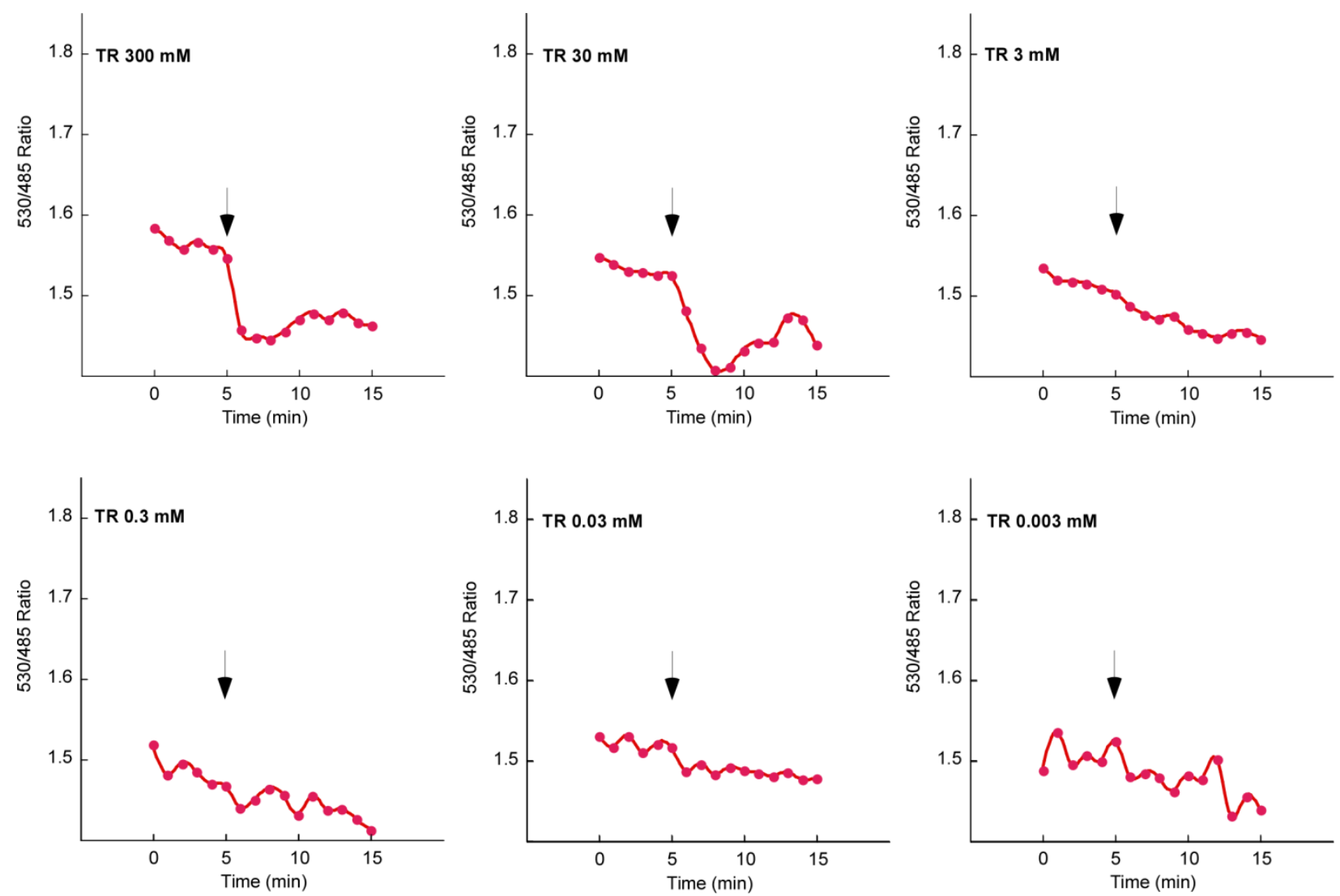

Figure 7. Average FRET Response Curves to Varying Ribose Concentrations in TR Cells. Average FRET response curves with ribose addition shown with the arrow at ribose concentrations ranging from $300 \mathrm{mM}$ to $0.003 \mathrm{mM}$.

We tested further concentrations of ribose to generate a saturation curve that produced a $K_{d}$ of $23.3 \mathrm{mM}$ ribose (Fig. 8). The $K_{d}$ value of recombinant Sil3-CRY protein isolated from $E$. coli was 142.84 uM (personal communication, K.E. Marshall), which was not significantly different from the published value for the CRY protein [4]. This suggests that the CRY biosensor embedded in diatom biosilica is 160 times less sensitive than the isolated protein. 

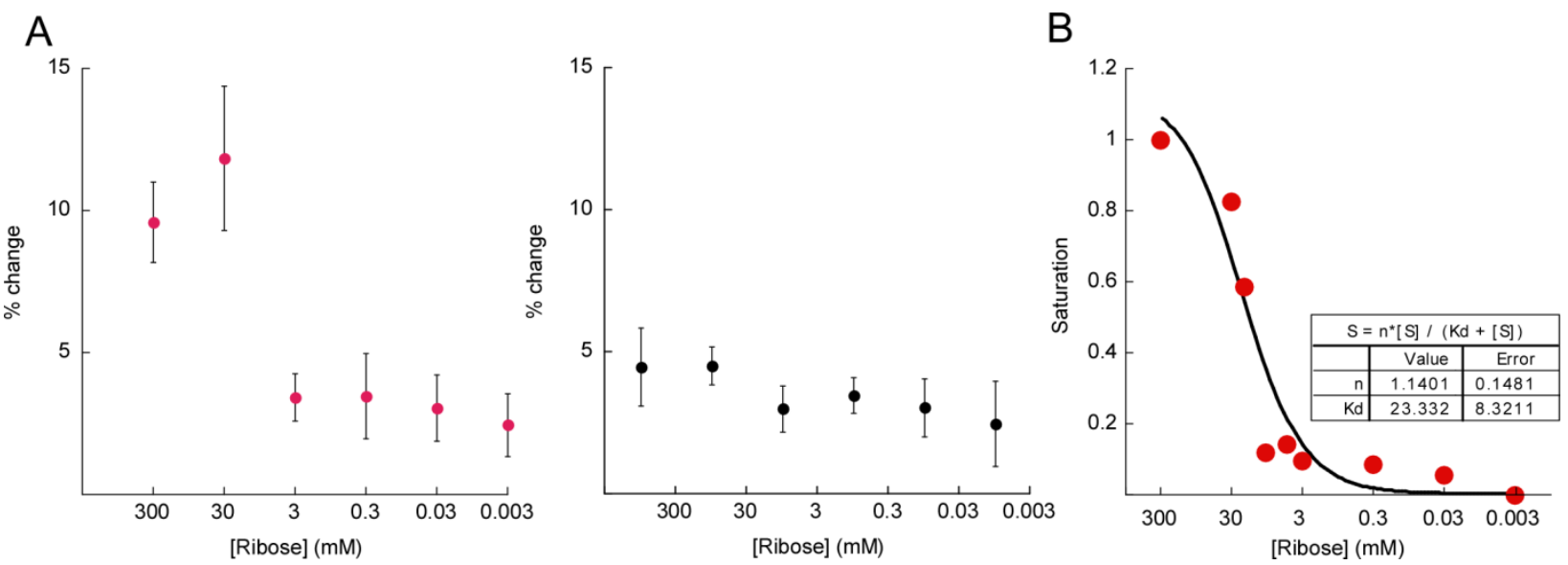

Figure 8. Average Percent FRET Change to Varying Ribose Concentrations and Saturation Curve. A) The average percent decrease in FRET response of TR (left) and WT (right) cells at various ribose concentrations. B) The saturation curve of TR cells produced a $K_{d}$ of $23.3 \mathrm{mM}$.

\section{DISCUSSION}

The goal of this research was to examine the FRET response of transformed diatom cells to external ribose concentrations and compare various transformations systems to determine the optimal vector configuration. We found TR cells to have statistically changes in FRET, which could be described by a saturation curve with a $K_{d}$ of $23.3 \mathrm{mM}$ ribose. In addition, we found that the gDNA one-vector system produced the largest and most consistent FRET response compared to a cDNA one-vector system or two-vector system.

The gDNA version was found to yield a more stable FRET response to ribose addition than the cDNA version. This may be due to cellular post-processing of the Sil3-CRY construct causing redirected localization of the protein, thereby resulting in a variable FRET response. The gDNA Sil3 version was originally used in the development of this transformation system, although that study does not go into detail as to why this version was used [4]. Perhaps it is 
because other investigators realized the cDNA version of Sil3 does not function as well as the gDNA version of Sil3 as we have seen in this study.

A one-vector system was determined to produce more consistent results than the twovector system. The one-vector system ensures that all cells resistance to nourseothricin will also contain the expression construct, eliminating the risk of having a mixed culture of diatoms that are antibiotic resistant but do not express the sensing construct. A two-vector system has been successful in previous work with $80 \%$ of the cells receiving both the antibiotic resistance vector and the expression cassette [3]. Our results were not as successful as less than half of the cells selected randomly showed the characteristic FRET curve. This suggests that less than half of the antibiotic resistant cells also correctly expressed the modified ribose receptor. This could be due to the size of the vector, the complexity of the protein, or the decrease in transformation efficiency of two vectors.

The sensitivity of the receptor sensor in living diatoms was several orders of magnitude less sensitive than the E. coli-isolated protein. This decrease in sensitivity could be due to the location of the protein in the biosilica. Previous research has shown that proteins directed to the biosilica are at least partially protected because they are embedded in the biosilica matrix [1]. Therefore, ribose movement could be hindered, reducing the concentration near the receptor. In addition, the ribose concentration could also be reduced as the diatoms use ribose in their natural cellular processes, and the exact ribose concentration is unknown when it reaches the receptor complex.

This study supports the use of diatom biology in application materials science. Verifying the functionality of biosilica-immobilized sensors demonstrates the possibility of inserting 
complex proteins into biosilica through "green synthesis" for biosensor development. Silica can be used as a platform for other applications as this method does not denature the protein as other methods do. Future directions include replacing the ribose binding protein with receptors for pollutants or enzymes required in organic synthesis, remediation and drug delivery.

\section{Acknowledgements}

The research presented was supported by the Marine Sciences Laboratory of Pacific Northwest National Laboratory and the U.S. Department of Energy through the Science Undergraduate Laboratory Internship. I would like to thank my mentors Dr. Guri Roesijadi and Dr. Kathryn Marshall for their guidance. Also, thank you to Carly Fairbanks who greatly supported and aided my work.

\section{References}

[1] Poulsen, N. et al, "Silica Immobilization of an Enzyme through Genetic Engineering of the Diatom Thalassiosira pseudonana,." Angew. Chem. Int. Ed., vol 46, pp. 1843-1846, 2007.

[2] Bozarth, A. et al, "Diatoms in Biotechnology: Modern Tools and Applications," Appl Microbiol Biotechnol, vol 82, pp 195-201, 2008.

[3] Poulsen, N. et al, "Molecular Genetic Manipulation of the Diatom Thalassiosira Pseudonana (Bacillariophyceae)," J Phycol, vol. 42, pp. 1059-1065, 2006.

[4] Lager, I. et al, "Development of a fluorescent nanosensor for ribose," FEBS, vol. 553, pp. $85-89,2003$. 
Filename: Diatom Biosensor_SULI_HMiller.docx

Directory: $\quad$ C: $:$ Documents and Settings $\backslash$ d3h082 $\backslash$ Local Settings $\backslash$ Temporary

Internet Files

Template: $\quad$ C: $\backslash$ Documents and Settings $\backslash$ 33h082\Application

Data $\backslash$ Microsoft $\backslash$ Templates $\backslash$ Normal.dotm

Title: $\quad$ FRET Response of a Modified Ribose Receptor Transformed in

Thalassiosira pseudonana

Subject:

Author: Kathryn Marshall

Keywords:

Comments:

Creation Date: $\quad$ 8/16/2011 2:19:00 PM

Change Number: 2

Last Saved On: $\quad$ 8/16/2011 2:19:00 PM

Last Saved By: $\quad$ Staff Member

Total Editing Time: 0 Minutes

Last Printed On: $\quad$ 1/5/2012 2:53:00 PM

As of Last Complete Printing

Number of Pages: 17

Number of Words: $\quad 3,537$ (approx.)

Number of Characters: 20,166 (approx.) 